\title{
The feasibility of diaphragmatic transplantation as potential therapy for treatment of respiratory failure associated with Duchenne muscular dystrophy: Acute canine model
}

\author{
Alexander Sasha Krupnick, MD, Andrew E. Gelman, PhD, Mikio Okazaki, MD, PhD, Jiaming Lai, MD, Nitin Das, MD, \\ Seiichiro Sugimoto, MD, Thomas H. Tung, MD, Steven B. Richardson, BS, G. Alexander Patterson, MD, and Daniel Kreisel, MD, \\ $\mathrm{PhD}$, St Louis, Mo
}

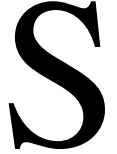

ince its introduction several decades ago, solid organ transplantation has had a wide impact as successful therapy for end-stage organ failure. Refinements in surgical techniques have allowed for the expansion of the spectrum of transplantation to replacement of "nontraditional" organs and tissue such as extremity limbs, facial structures, and laryngeal allografts. ${ }^{1}$ Duchenne muscular dystrophy (DMD) is the most common and severe of the human muscular dystrophies, affecting 1 in 3500 live male births. Currently no efficacious therapy is available for this disease, and death generally occurs in the late teens to early twenties from respiratory complications resulting from diaphragmatic dysfunction. ${ }^{2}$ Diaphragm transplantation could thus represent a novel therapy for this disease. As the ultimate success of transplantation hinges on adequate revascularization, the goal of this study was to develop surgical techniques for orthotopic diaphragmatic transplantation.

\section{Methods, Results and Discussion}

The canine model has been used to develop multiple surgical techniques in thoracic organ transplantation, and the experiments described herein were performed in mongrel hounds weighing 25 to 30 pounds. Similar to human beings, the phrenic arteries in the dog branch directly off the abdominal aorta and are reported to act as the main source of blood supply ${ }^{3}$ to the crural and costal diaphragm. To evaluate the possibility of diaphragmatic survival as a free flap based on the phrenic vessels alone, we removed collateral diaphragmatic blood flow by ligating the intercostal branches, internal thoracic arteries, and epigastric and abdominal wall vessels. We then examined regional distribution of blood flow to the diaphragm with multicolored fluorescent microspheres (IMT Labs, Irvine, Calif). This was accomplished by injecting $25 \times 10^{6}$ microspheres of different colors into the left atrium after ligation of collaterals. A ref-

From the Department of Surgery, Division of Cardiothoracic Surgery, Thoracic Immunobiology Laboratory, Division of Plastic and Reconstructive Surgery, Washington University in St. Louis.

Supported in part by R21 HL081240-01 GAP.

Received for publication Sept 27, 2007; accepted for publication Dec 4, 2007.

Address for reprints: Alexander Sasha Krupnick, MD, Washington University Division of Cardiothoracic Surgery, 660 So Euclid, Campus Box 8234, St. Louis, MO 63110 (E-mail: krupnicka@wudosis.wustl.edu).

J Thorac Cardiovasc Surg 2008;135:1398-9

$0022-5223 / \$ 34.00$

Copyright (C) 2008 by The American Association for Thoracic Surgery doi:10.1016/j.jtcvs.2007.12.047 erence blood sample was withdrawn from the femoral artery. For evaluation of regional blood flow, the right and left hemidiaphragms were divided into 3 regions designated as zone 1, costal diaphragm furthest from the phrenic blood supply, zone 2, costal diaphragm closest to the phrenic vessels, and zone 3 , the crural diaphragm. The number of microbeads in each sample and the reference blood were counted by flow cytometry after tissue digestion and the regional blood flow determined by the following equation: $Q i(\mathrm{~mL} / \mathrm{min})=\left(f l_{i} / f l_{r e f}\right) \cdot R(\mathrm{~mL} / \mathrm{min})$, where $Q i=$ flow to the tissue, $\mathrm{fl}_{\mathrm{i}}=$ fluorescence count in each tissue, $f l_{\text {ref }}=$ fluorescence count of the reference blood sample, and $R=$ the reference blood withdrawal rate (Figure E1). Total as well as regional blood flow in the crural and costal diaphragm did not decrease after ligation of these collaterals, supporting our hypothesis that the diaphragm can be transplanted as a free flap based on the phrenic vessels (Figure E1).

We then proceeded with orthotopic transplantation. Owing to the anatomic difficulty of transplanting the whole canine diaphragm, it was decided for the purpose of model development to transplant the left hemidiaphragm. The donor graft was harvested by serial ligation of all collateral vasculature except phrenic branches off the aorta and phrenic venous tributaries to the inferior vena cava. The abdominal and chest wall attachments were separated and the costal and crural diaphragm was removed along with a rim of costal margin (Figure 1, $A$ ). The graft was topically cooled to $4^{\circ} \mathrm{C}$. Initial attempts to implant the donor aortic and vena caval cuffs as interposition grafts in the recipient with a period of complete aortic occlusion resulted in high operative mortality. We were then successful in developing methods of transplantation consisting of dual end-to-side donor-to-recipient suprahepatic inferior vena cava anastomosis using a partially occlusive side-biter clamp (Figure 1, $B)$. Arterial inflow was provided by an end-to-side anastomosis of the donor to the recipient aorta using a similar technique (Figure 1, $C)$. The left hemidiaphragm of the recipient was excised and the transplanted graft inserted orthotopically by securing it to surrounding structures. Statistically identical blood flow was documented in the transplanted left hemidiaphragm compared with the native left hemidiaphragm of control animals 30 minutes after reperfusion (n $=5$ ) (2-tailed $t$ test of probability) (Figure 2).

\section{Discussion}

To our knowledge, this is the first demonstration of technical feasibility of diaphragmatic transplantation. We are in the process of establishing a long-term transplantation model that will allow us to study physiology, immunosuppression, and long-term survival and metabolic requirements of a transplanted diaphragm. Methods for reinnervation or direct diaphragmatic pacing will be explored. 


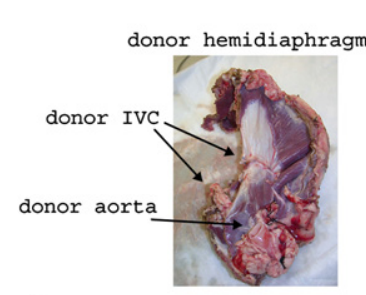

A

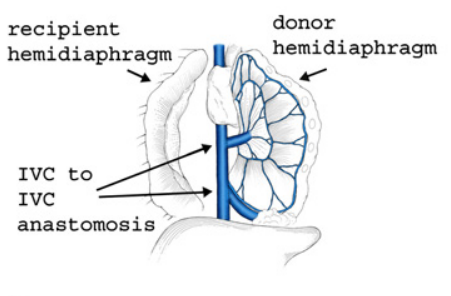

B

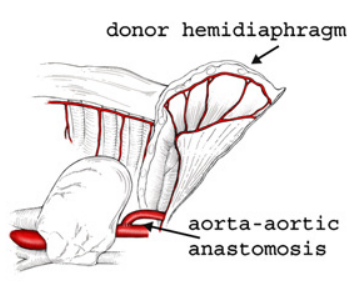

C
Figure 1. Donor hemidiaphragm after harvest (A). Heterotopic allogenic transplantation was performed by dual end-to-end anastomosis of suprahepatic and infrahepatic donor IVC to recipient IVC (B) and arterial inflow was established by a single aorta-aortic anastomosis (C). IVC, Inferior vena cava.
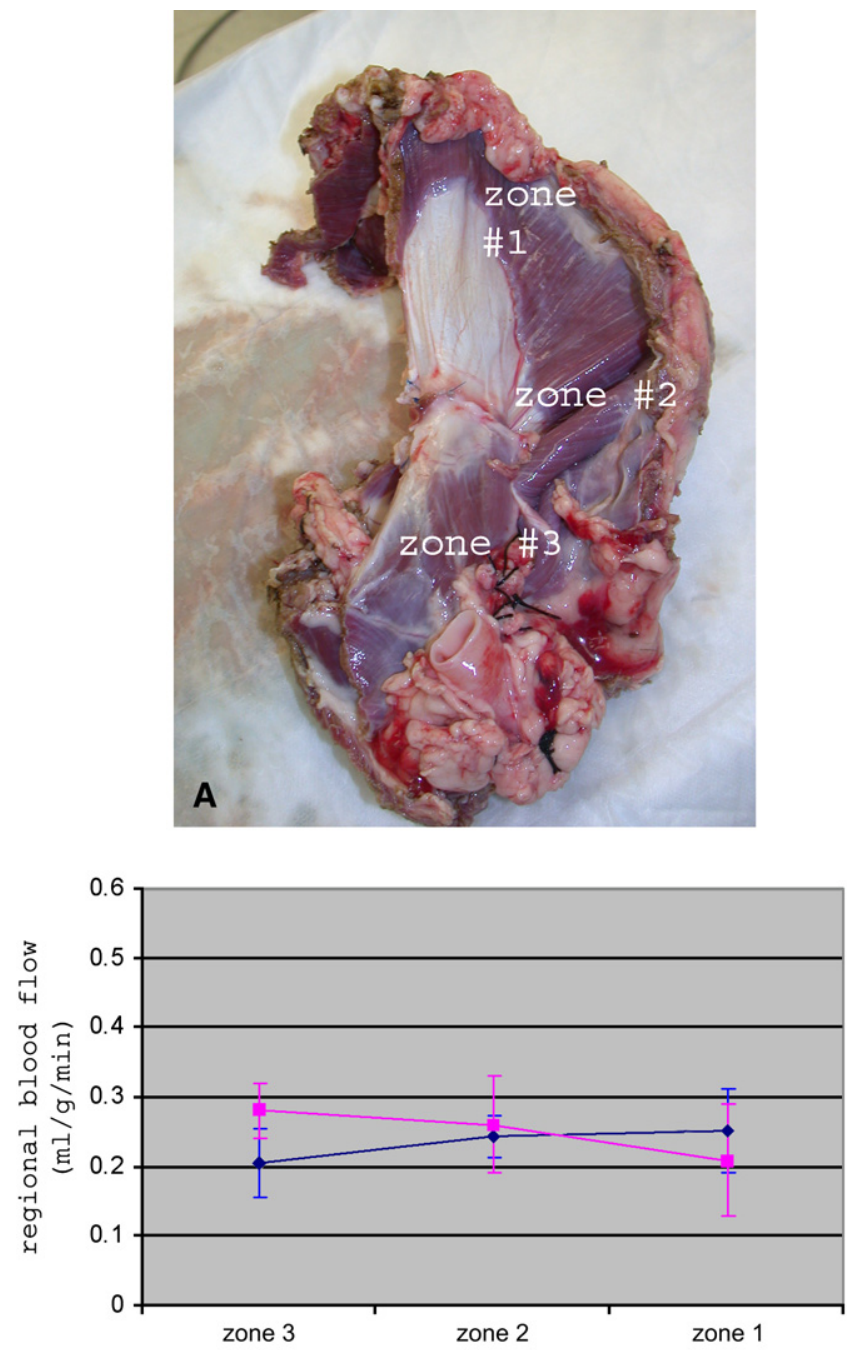

Blood flow to the transplant

B

Native blood flow

Figure 2. After orthotopic transplantation, blood flow was statistically identical to that of the native, unmanipulated diaphragm of control animals in all zones. Black line, Native unmanipulated diaphragm; purple line, transplanted diaphragm) (5 animals represented).
Inasmuch as a homolog of DMD has been identified in a muscular dystrophic Golden Retriever, the canine model may provide an opportunity for future preclinical large animal trials.

\section{References}

1. Tobin GR, Breidenbach WC 3rd, Pidwell DJ, Ildstad ST, Ravindra KV. Transplantation of the hand, face, and composite structures: evolution and current status. Clin Plast Surg. 2007;34:271-8, ix-x.

2. Smith PE, Calverley PM, Edwards RH, Evans GA, Campbell EJ. Practical problems in the respiratory care of patients with muscular dystrophy. N Engl J Med. 1987;316:1197-205.

3. Plestis K, Fell SC. The diaphragm: anatomy, embryology, pathophysiology, and surgery of the phrenic nerve and diaphragm. In: Pearson F, Cooper J, Deslauriers J, Ginsberg RJ, Hiebert G, eds. Philadelphia: Churchill Livingstone; 2002:1499. 

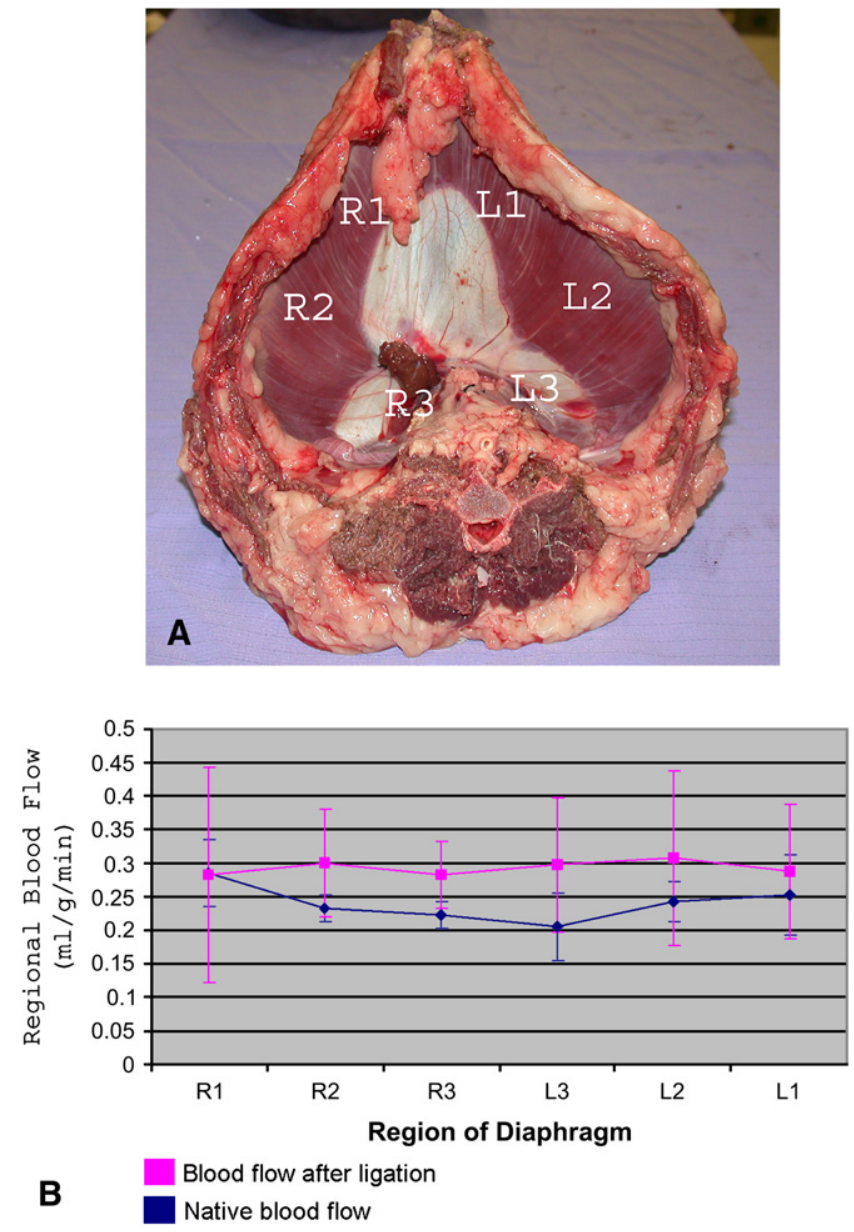

Figure E1. Top panel is a visual representation of the different zones of the diaphragm with zone 1 representing the costal ventral diaphragm, zone 2 the costal dorsal diaphragm, and zone 3 the crural diaphragm ( $R 1$, Right zone 1; $R 2$, right zone 2; $R 3$, right zone 3; $L 1$, left zone $1 ; \boldsymbol{L}$, left zone $2 ; \boldsymbol{L}$, left zone 3 ). Bottom panel is a graphic representation of regional blood flow after ligation of all collaterals except direct phrenic branches. Blood flow to all zones of the diaphragm remained statistically identical after ligation of all collateral vessels. Black line, Preligation; purple line, postligation (4 animals represented). 